\title{
Almost sure synchronization control for stochastic delayed complex networks based on pinning adaptive method
}

\author{
Tianbo Wang ${ }^{1 *}$, Shouwei Zhao', Wuneng Zhou ${ }^{2}$ and Weiqin Yu ${ }^{1}$
}

\section{"Correspondence:}

tb_wang@163.com

${ }^{1}$ College of Fundamental Studies,

Shanghai University of Engineering

Science, Longteng Road, Shanghai,

P.R. China

Full list of author information is

available at the end of the article

\begin{abstract}
This paper investigates the almost sure synchronization control problem for a class of stochastic delayed complex networks by using the stochastic differential equation theory and the Kronecker product technique. Different from the existing works, the considered problem is that all the nodes in the complex networks can synchronize with each other although the target node is unknown. Some sufficient conditions which guarantee the complex networks to have almost sure synchronization are derived and two kinds of controllers are designed, respectively. Finally, a numerical example is given to illustrate the effectiveness of the main results.
\end{abstract}

Keywords: complex network; almost sure synchronization; stochastic disturbance; pinning adaptive control

\section{Introduction}

Complex dynamical networks are composed of a family of interconnected nodes, in which each node denotes an individual element in the network and adjusts its behavior by the information received from its neighbor nodes. They can be used to model some complex nonlinear dynamical systems in science and engineering. Thus, in recent years, complex networks have attracted increasing attention in various fields such as biology [1,2], sociology [3], and physics $[4,5]$.

In the dynamical behaviors of complex networks, synchronization motion is one of the important elements. Synchronization means that all the nodes' action in complex networks will attain the same dynamic behavior along with the time evolution. For example, both a group of fish swarming together and a flock of birds synchronously flying belong to the synchronization phenomena. Up to now, there exists much literature such as [627 ] studying the synchronization control problem of complex networks by using different methods. For instance, $[6,7]$ study the synchronization control problem of discrete complex dynamical networks with a time varying delay by using the method of partitioning time delay and chief stability function, respectively. For the continuous complex networks with different characters, such as time delayed complex networks [8-10], stochastic complex networks [11, 12], complex networks with switching topology [13-15], there have existed a great deal of papers to study the synchronization control problem. The control methods mainly include pinning control [16-18], impulsive control [19, 20], adaptive con-

(c) Wang et al. 2016. This article is distributed under the terms of the Creative Commons Attribution 4.0 International License (http://creativecommons.org/licenses/by/4.0/), which permits unrestricted use, distribution, and reproduction in any medium, provided you give appropriate credit to the original author(s) and the source, provide a link to the Creative Commons license, and indicate if changes were made. 
trol [21-27], etc. The adopted theories mainly include Lyapunov stability theory, the chief stability function method, and $M$-matrix theory.

It should be noted that most of these works required all the nodes in complex networks to synchronize with the target node or the isolated node given beforehand. If the target node is unknown, then these results and methods could fail to achieve the synchronization because the designed controllers are usually based on the state information of the target node. In fact, when the target node is known, each node in the complex networks could adjust its behavior according to the error with the target node. However, if the target node is unknown, each node can only adjust its behavior according to the information from its adjacent nodes, at the same time, the adjacent nodes are varying. Practically, the phenomena of the unknown target node also exist in the real world. For example, for a complex network consisting of some multi-agents without leader, all the agents achieve consensus by adjusting the information received from its adjacent agents. In addition, sometimes it is difficult to precisely describe the state equation of the target node when systems are disturbed by stochastic noise and transmission time delay. Hence, it is necessary to analyze the synchronization control problem of complex networks with unknown target node.

On the other hand, the stochastic complex networks models are very common and there also exists much literature such as [11,12] and [24-27] studying synchronization problems of stochastic complex network. However, these papers mainly focus on the synchronization in mean square. For the almost sure synchronization of complex networks, there exist few results. Especially, the complex network which is the almost sure synchronization could not have synchronization in mean square, the relative counter-example can be found in $[28-30]$.

Motivated by the above discussion, in this paper, we will consider the almost sure synchronization control problem for a class of stochastic delayed complex networks. The contributions of our paper are as follows. (i) The almost sure synchronization control other than synchronization in mean square is investigated. (ii) The provided results can suit for the synchronization of complex networks with the target node unknown. (iii) The obtained results only depend on the complex network's parameters.

The rest of this paper is organized as follows. In Section 2, we introduce the stochastic delayed complex dynamical network model and some useful lemmas. In Section 3, some criteria which ensure that the complex network synchronizes well are derived and some synchronization controllers are given. In Section 4, a numerical example is provided to illustrate the effectiveness of our proposed results. Finally, this paper ends with conclusions in Section 5.

Notation $R^{n}$ and $R^{n \times m}$ denote the $n$-dimensional Euclidean space and the set of all $n \times m$ dimensional real matrices, respectively. For a vector $v=\left(v_{1}, v_{2}, \ldots, v_{n}\right)^{T} \in R^{n}$, whose 2norm is denoted by $\|v\|_{2}=\sqrt{\sum_{i=1}^{n} v_{i}^{2}} \cdot A^{T}, \operatorname{tr}(A)$, and $\operatorname{det}(A)$ represent the transpose, trace, and determinant of the matrix $A$, respectively. $\lambda_{\min }(A)$ and $\lambda_{\max }(A)$ represent the minimum and maximum eigenvalues of the matrix $A$, respectively. $X \geq Y$ (respectively, $X>Y$ ) means that $X-Y$ is a symmetric positive semi-definite matrix (respectively, positive definite matrix), where $X, Y$ are symmetric matrices. $I_{n}$ is the $n \times n$ identity matrix, $\otimes$ is the Kronecker product. The set $\left(\Omega, \mathcal{F},\left\{\mathcal{F}_{t}\right\}_{t \geq 0}, \mathcal{P}\right)$ denotes the complete probability space with a filtration $\left\{\mathcal{F}_{t}\right\}_{t \geq 0}$ satisfying right continuity and $\mathcal{F}_{0}$ containing all $\mathcal{P}$-null sets. $C_{\mathcal{F}_{0}}^{b}\left([-\tau, 0] ; R^{n}\right)$ denotes the family of all bounded $\mathcal{F}_{0}$-measurable $C\left([-\tau, 0] ; R^{n}\right)$ valued random variables. Throughout this paper, all matrices have the appropriate dimensions. 


\section{Problem formulation and preliminaries}

In this paper, we consider the following stochastic delayed complex network composed of $N$ identical nodes with linear couplings. Each node is an $n$-dimensional dynamical subsystem, whose state equation is described by

$$
\left\{\begin{aligned}
& d x_{i}(t)= {\left[A x_{i}(t)+f\left(t, x_{i}(t), x_{i}(t-\tau)\right)+\sum_{j=1}^{N} c_{i j} \Gamma x_{j}(t-\tau)+u_{i}(t)\right] d t } \\
&+g\left(x_{i}(t)\right) d w(t), \quad i=1,2, \ldots, N \\
& x_{i}(t)=\psi_{i}(t), \quad t \in[-\tau, 0],
\end{aligned}\right.
$$

where $N$ is the number of coupled nodes. $x_{i}(t)=\left(x_{i 1}(t), x_{i 2}(t), \ldots, x_{i n}(t)\right)^{T} \in R^{n}$ denotes the state vector, $A \in R^{n \times n}$ is a constant real matrix, $f(\cdot) \in R^{n}$ is a continuous differential vector function. $C=\left[c_{i j}\right] \in R^{N \times N}$ is the outer-coupling matrix, where $c_{i j}$ is defined as follows: if there exists a connection between node $i$ with node $j$, then $c_{i j}>0$; otherwise, $c_{i j}=0$. In addition, the elements of the matrix $C$ satisfy

$$
c_{i i}=-\sum_{j=1, j \neq i}^{N} c_{i j}
$$

$\Gamma \in R^{n \times n}$ is the inner-coupling matrices, $\tau>0$ denotes the transmission time delay, $u_{i}(t) \in$ $R^{n}$ is the control input to be designed in the sequel. $w(t)$ is a 1-dimensional Brownian motion defined on the probability space $\left(\Omega, \mathcal{F},\left\{\mathcal{F}_{t}\right\}_{t \geq 0}, \mathcal{P}\right)$ with

$$
E\{w(t)\}=0, \quad E\left\{d w^{2}(t)\right\}=d t
$$

$g(\cdot) \in R^{n}$ is the noise intensity. The initial condition $\psi_{i}(t) \in R^{n}$ is a continuous vector function.

Definition 1 Complex network (1) is said to have almost sure synchronization if

$$
\lim _{t \rightarrow+\infty}\left(x_{i}(t)-x_{j}(t)\right)=0, \quad \text { a.s. }
$$

holds for $i, j=1,2, \ldots, N$.

Remark 1 The idea of Definition 1 comes from [28-30]. It is obvious that complex network (1) can achieve almost sure synchronization only if

$$
\lim _{t \rightarrow+\infty}\left(x_{i}(t)-x_{1}(t)\right)=0, \quad \text { a.s. }
$$

holds for $i=2, \ldots, N$. Compared with the synchronization in mean square for complex networks [24-30], the almost sure synchronization is more general, the detailed difference for this two concepts can be found in [30]. On the other hand, it should be noticed that the theory used in this paper is similar to [31]. But the proposed results and methods in [31] are not suitable for this paper. This paper considers a complex network with the unknown target node other than a master-slave system.

In order to study the almost sure synchronization of complex network (1), we can arbitrarily choose a node as the target node. Without the loss of generality, we assume that the 
state of the target node is $x_{1}(t)$. Let $e_{i}(t)=x_{i}(t)-x_{1}(t)$, then one gets the error system as follows:

$$
\left\{\begin{aligned}
d e_{i}(t)= & {\left[A e_{i}(t)+f\left(t, x_{i}(t), x_{i}(t-\tau)\right)-f\left(t, x_{1}(t), x_{1}(t-\tau)\right)\right.} \\
& \left.+\sum_{j=1}^{N}\left(c_{i j}-c_{1 j}\right) \Gamma e_{j}(t-\tau)+u_{i}(t)-u_{1}(t)\right] d t \\
& +\left[g\left(x_{i}(t)\right)-g\left(x_{1}(t)\right)\right] d w(t), \quad i=2, \ldots, N, \\
e_{i}(t)= & \psi_{i}(t)-\psi_{1}(t), \quad t \in[-\tau, 0] .
\end{aligned}\right.
$$

Writing $e(t)=\left(e_{2}^{T}(t), e_{3}^{T}(t), \ldots, e_{N}^{T}(t)\right)^{T}$,

$$
\begin{aligned}
& F_{i}\left(t, e_{i}(t), e_{i}(t-\tau)\right)=f\left(t, x_{i}(t), x_{i}(t-\tau)\right)-f\left(t, x_{1}(t), x_{1}(t-\tau)\right), \\
& F(t, e(t), e(t-\tau))=\left(F_{2}^{T}\left(t, e_{2}(t), e_{2}(t-\tau)\right), \ldots, F_{N}^{T}\left(t, e_{N}(t), e_{N}(t-\tau)\right)\right)^{T}, \\
& G(e(t))=\left(g^{T}\left(x_{2}(t)\right)-g^{T}\left(x_{1}(t)\right), \ldots, g^{T}\left(x_{N}(t)\right)-g^{T}\left(x_{1}(t)\right)\right)^{T}, \\
& U(t)=\left(u_{2}^{T}(t)-u_{1}^{T}(t), \ldots, u_{N}^{T}(t)-u_{1}^{T}(t)\right)^{T}, \\
& \Psi(t)=\left(\psi_{2}^{T}(t)-\psi_{1}^{T}(t), \ldots, \psi_{N}^{T}(t)-\psi_{1}^{T}(t)\right)^{T}, \\
& \tilde{C}=\left[\begin{array}{ccc}
c_{22}-c_{12} & \cdots & c_{2 N}-c_{1 N} \\
\vdots & \cdots & \vdots \\
c_{N 2}-c_{12} & \cdots & c_{N N}-c_{1 N}
\end{array}\right] \in R^{(N-1) \times(N-1),}
\end{aligned}
$$

thus error system (2) can be written in the following compact form:

$$
\left\{\begin{aligned}
d e(t)= & {\left[\left(I_{N-1} \otimes A\right) e(t)+F(t, e(t), e(t-\tau))\right.} \\
& +(\tilde{C} \otimes \Gamma) e(t-\tau)+U(t)] d t+G(e(t)) d w(t) \\
e(t)= & \Psi(t), \quad t \in[-\tau, 0]
\end{aligned}\right.
$$

Remark 2 Different from [16-18], it is not necessary to exactly know the state equation of the target node

$$
\dot{s}(t)=A s(t)+f(s(t))
$$

in this paper. Hence, the methods in these papers are not suitable for our paper.

Before moving on, we present some necessary assumptions and lemmas.

(H1) Assume that there exist two constants $M_{1} \geq 0$ and $M_{2} \geq 0$ such that

$$
\begin{aligned}
& \left\|f\left(t, \xi_{1}(t), \xi_{1}(t-\tau)\right)-f\left(t, \xi_{2}(t), \xi_{2}(t-\tau)\right)\right\|^{2} \\
& \quad \leq M_{1}\left\|\xi_{1}(t)-\xi_{2}(t)\right\|^{2}+M_{2}\left\|\xi_{1}(t-\tau)-\xi_{2}(t-\tau)\right\|^{2}
\end{aligned}
$$

for any $\xi_{1}(t), \xi_{2}(t) \in R^{n}$ and $t>0$.

(H2) Assume that there exists a constant $L \geq 0$ such that

$$
\left\|g\left(\xi_{1}(t)\right)-g\left(\xi_{2}(t)\right)\right\|^{2} \leq L\left\|\xi_{1}(t)-\xi_{2}(t)\right\|^{2}
$$

for any $\xi_{1}(t), \xi_{2}(t) \in R^{n}$ 
Lemma 1 ([32]) The matrix $C$ has a single eigenvalue 0 and all the other eigenvalues are negative.

Remark 3 Without loss of generality, in this paper, we assume that the eigenvalues of the matrix $C$ are $0=\lambda_{1} \geq \lambda_{2} \geq \lambda_{3} \geq \cdots \geq \lambda_{N}$.

Lemma 2 The eigenvalues of the matrix $C$ are composed of the eigenvalues of matrix $\tilde{C}$ and 0 .

Proof According to the definition of the eigenvalue and properties of the determinant, we have

$$
\begin{aligned}
\operatorname{det}\left(\lambda I_{N}-C\right) & =\operatorname{det}\left[\begin{array}{cccc}
\lambda-c_{11} & -c_{12} & \cdots & -c_{1 N} \\
-c_{21} & \lambda-c_{22} & \cdots & -c_{2 N} \\
\vdots & \vdots & \vdots & \vdots \\
-c_{N 1} & -c_{N 2} & \cdots & \lambda-c_{N N}
\end{array}\right] \\
& =\operatorname{det}\left[\begin{array}{cccc}
\lambda & -c_{12} & \cdots & -c_{1 N} \\
\lambda & \lambda-c_{22} & \cdots & -c_{2 N} \\
\vdots & \vdots & \vdots & \vdots \\
\lambda & -c_{N 2} & \cdots & \lambda-c_{N N}
\end{array}\right] \\
& =\lambda \cdot \operatorname{det}\left[\begin{array}{cccc}
1 & -c_{12} & \cdots & -c_{1 N} \\
1 & \lambda-c_{22} & \cdots & -c_{2 N} \\
\vdots & \vdots & \vdots & \vdots \\
1 & -c_{N 2} & \cdots & \lambda-c_{N N}
\end{array}\right] \\
& =\lambda \cdot \operatorname{det}\left[\begin{array}{cccc}
1 & -c_{12} & \cdots & -c_{1 N} \\
0 & \lambda-\left(c_{22}-c_{12}\right) & \cdots & -\left(c_{2 N}-c_{1 N}\right) \\
\vdots & \vdots & \vdots & \vdots \\
0 & -\left(c_{N 2}-c_{12}\right) & \cdots & \lambda-\left(c_{N N}-c_{1 N}\right)
\end{array}\right] \\
& =\lambda \cdot \operatorname{det}\left(\lambda I_{N-1}-\tilde{C}\right) .
\end{aligned}
$$

Therefore, one more eigenvalue of matrix $C$ than $\tilde{C}$ is the single 0 . The proof is completed.

From Lemma 2, we know that the eigenvalues of the matrix $\tilde{C}$ are $\lambda_{2}, \lambda_{3}, \ldots, \lambda_{N}$, respectively.

Lemma 3 ([33]) Assume that the stochastic differential delay equation

$$
d x(t)=f(x(t), x(t-\tau), t) d t+g(x(t), x(t-\tau), t) d v(t)
$$

exists a unique solution $x(t, \xi(t))$ on $t \geq 0$ for any given initial condition $\xi(t) \in C_{\mathcal{F}_{0}}^{b}([-\tau, 0]$; $\left.R^{n}\right)$, where $v(t)$ is a $n$-dimensional Wiener process. Moreover, both $f(x(t), x(t-\tau), t)$ and $g(x(t), x(t-\tau), t)$ are locally bounded and uniformly bounded on $t$. If there exist functions 


$$
\begin{aligned}
& V(x(t), t) \in C^{2,1}\left(R^{n} \times R_{+} ; R_{+}\right), \beta(t) \in L^{1}\left(R_{+} ; R_{+}\right) \text {and } \omega_{1}, \omega_{2} \in C\left(R^{n} ; R_{+}\right) \text {such that } \\
& \mathcal{L} V(x(t), x(t-\tau), t) \leq \beta(t)-\omega_{1}(x(t))+\omega_{2}(x(t-\tau)), \quad \forall x(t) \in R^{n}, \\
& \omega_{1}(x(t))>\omega_{2}(x(t)), \quad \forall x(t) \neq 0, \\
& \lim _{\|x\| \rightarrow \infty} \inf _{0 \leq t<\infty} V(x(t), t)=\infty,
\end{aligned}
$$

then

$$
\lim _{t \rightarrow \infty} x(t, \xi(t))=0, \quad \text { a.s. }
$$

for every $\xi(t) \in C_{\mathcal{F}_{0}}^{b}\left([-\tau, 0] ; R^{n}\right)$, where the operator $\mathcal{L} V(x(t), t)$ is defined as

$$
\begin{aligned}
\mathcal{L} V(x(t), t)= & V_{t}(x(t), t)+V_{x}(x(t), t) f(x(t), x(t-\tau), t) \\
& +\frac{1}{2} \operatorname{tr}\left\{g^{T}(x(t), x(t-\tau), t) V_{x x} g(x(t), x(t-\tau), t)\right\},
\end{aligned}
$$

$V_{t}(x(t), t)=\frac{\partial V(x(t), t)}{\partial t}, V_{x}(x(t), t)=\left(\frac{\partial V(x(t), t)}{\partial x_{1}}, \frac{\partial V(x(t), t)}{\partial x_{2}}, \ldots, \frac{\partial V(x(t), t)}{\partial x_{n}}\right)$ and $V_{x x}=\left[\frac{\partial^{2} V(x(t), t)}{\partial x_{i} \partial x_{j}}\right]_{n \times n}$

Lemma 4 ([34]) The Kronecker product $\otimes$ has the following properties:

(1) $(A+B) \otimes C=A \otimes C+B \otimes C, C \otimes(A+B)=C \otimes A+C \otimes B$;

(2) $(A \otimes B)^{T}=A^{T} \otimes B^{T}$;

(3) $(A \otimes C)(B \otimes D)=A B \otimes C D$;

(4) $\lambda(A \otimes B)=\left\{\gamma_{i} \theta_{j}, i=1,2, \ldots, n, j=1,2, \ldots, m\right\}$,

where $A, B, C$, and $D$ are real matrices with appropriate dimensions, $\gamma_{i}(i=1,2, \ldots, n)$ are the eigenvalues of the matrix $A \in R^{n \times n}$ and $\theta_{j}(j=1,2, \ldots, m)$ are the eigenvalues of matrix $B \in R^{m \times m}$.

\section{Synchronization analysis and control}

\subsection{Synchronization analysis}

In this section, we will first analyze the almost sure stability of system (3) without control input, i.e.,

$$
\left\{\begin{array}{l}
\operatorname{de}(t)=\left[\left(I_{N-1} \otimes A\right) e(t)+F(t, e(t), e(t-\tau))+(\tilde{C} \otimes \Gamma) e(t-\tau)\right] d t+G(e(t)) d w(t), \\
e(t)=\Psi(t), \quad t \in[-\tau, 0]
\end{array}\right.
$$

Theorem 1 Suppose that assumptions (H1) and (H2) hold. If there exist positive definite symmetric matrices $P, R \in R^{n \times n}$ and positive constants $\alpha>0, \beta>0, \mu>0$, such that

$$
P<\mu I_{n}
$$

and

$$
\Theta_{1}=\left[\begin{array}{ccc}
\Theta_{1,11} & P & P \\
P & -\alpha I_{n} & 0 \\
P & 0 & -\beta I_{n}
\end{array}\right]<0
$$


where

$$
\Theta_{1,11}=P A+A^{T} P+\tau R+\left(M_{1} \alpha+M_{2} \alpha+\mu L\right) \cdot I_{n}+\beta \lambda_{N}^{2} \Gamma^{T} \Gamma,
$$

then system (8) is almost sure asymptotic stability for any initial condition.

Proof Choose the following Lyapunov function:

$$
V(e(t))=e^{T}(t)\left(I_{N-1} \otimes P\right) e(t)+\int_{-\tau}^{0} \int_{t+s}^{t} e^{T}(\theta)\left(I_{N-1} \otimes R\right) e(\theta) d \theta d s
$$

By Lemma 3, the differential of $V(e(t))$ along the state trajectories of system (8) is

$$
d V(e(t))=\mathcal{L} V(e(t)) d t+2 e^{T}(t)\left(I_{N-1} \otimes P\right) G(e(t)) d w(t)
$$

where

$$
\begin{aligned}
\mathcal{L} V(e(t))= & 2 e^{T}(t)\left(I_{N-1} \otimes P\right)\left[\left(I_{N-1} \otimes A\right) e(t)+F(t, e(t), e(t-\tau))\right. \\
& +(\tilde{C} \otimes \Gamma) e(t-\tau)]+\operatorname{tr}\left\{G^{T}(e(t))\left(I_{N-1} \otimes P\right) G(e(t))\right\} \\
& +\tau e^{T}(t)\left(I_{N-1} \otimes R\right) e(t)-\int_{t-\tau}^{t} e^{T}(s)\left(I_{N-1} \otimes R\right) e(s) d s \\
\leq & e^{T}(t)\left[I_{N-1} \otimes\left(P A+A^{T} P+\tau R\right)\right] e(t) \\
& +2 e^{T}(t)\left(I_{N-1} \otimes P\right) F(t, e(t), e(t-\tau)) \\
& +2 e^{T}(t)\left(I_{N-1} \otimes P\right)(\tilde{C} \otimes \Gamma) e(t-\tau) \\
& +\operatorname{tr}\left\{G^{T}(e(t))\left(I_{N-1} \otimes P\right) G(e(t))\right\} .
\end{aligned}
$$

From assumptions (H1)-(H2) and (9), for any $\alpha>0$, one gets

$$
\begin{aligned}
& 2 e^{T}(t)\left(I_{N-1} \otimes P\right) F(t, e(t), e(t-\tau)) \\
& =2 \sum_{i=2}^{N} e_{i}^{T}(t) P\left[f\left(t, x_{i}(t), x_{i}(t-\tau)\right)-f\left(t, x_{1}(t), x_{1}(t-\tau)\right)\right] \\
& \leq \sum_{i=2}^{N}\left\{\alpha^{-1} e_{i}^{T}(t) P P^{T} e_{i}(t)+\alpha F_{i}^{T}\left(t, e_{i}(t), e_{i}(t-\tau)\right) F_{i}\left(t, e_{i}(t), e_{i}(t-\tau)\right)\right\} \\
& \leq \alpha^{-1} e^{T}(t)\left(I_{N-1} \otimes P\right)\left(I_{N-1} \otimes P\right) e(t)+M_{1} \alpha e^{T}(t) e(t) \\
& \quad+M_{2} \alpha e^{T}(t-\tau) e(t-\tau)
\end{aligned}
$$

and

$$
\begin{aligned}
G^{T} & (e(t))\left(I_{N-1} \otimes P\right) G(e(t)) \\
\quad= & \sum_{i=2}^{N}\left[g\left(x_{i}(t)\right)-g\left(x_{1}(t)\right)\right]^{T} P\left[g\left(x_{i}(t)\right)-g\left(x_{1}(t)\right)\right]
\end{aligned}
$$




$$
\begin{aligned}
& \leq \lambda_{\max }(P) \sum_{i=2}^{N}\left[g\left(x_{i}(t)\right)-g\left(x_{1}(t)\right)\right]^{T}\left[g\left(x_{i}(t)\right)-g\left(x_{1}(t)\right)\right] \\
& \leq \lambda_{\max }(P) L \sum_{i=2}^{N} e_{i}^{T}(t) e_{i}(t) \\
& \leq \mu L e^{T}(t) e(t) .
\end{aligned}
$$

On the other hand, it is noted that the inequality

$$
\begin{aligned}
& 2 e^{T}(t)\left(I_{N-1} \otimes P\right)(\tilde{C} \otimes \Gamma) e(t-\tau) \\
& \quad \leq \beta^{-1} e^{T}(t)\left(I_{N-1} \otimes P\right)\left(I_{N-1} \otimes P\right) e(t) \\
& \quad+\beta e^{T}(t-\tau)(\tilde{C} \otimes \Gamma)^{T}(\tilde{C} \otimes \Gamma) e(t-\tau)
\end{aligned}
$$

holds for any positive constant $\beta>0$.

From (14)-(16) and Lemma 4, one gets

$$
\begin{aligned}
\mathcal{L} V(e(t)) \leq & e^{T}(t)\left\{I_{N-1} \otimes\left[P A+A^{T} P+\tau R+\left(M_{1} \alpha+\mu L\right) \cdot I_{n}\right]\right. \\
& \left.+\left(\alpha^{-1}+\beta^{-1}\right)\left(I_{N-1} \otimes P\right)\left(I_{N-1} \otimes P\right)\right\} e(t) \\
& +e^{T}(t-\tau)\left[M_{2} \alpha \cdot I_{n(N-1)}+\beta(\tilde{C} \otimes \Gamma)^{T}(\tilde{C} \otimes \Gamma)\right] e(t-\tau) . \\
= & -e^{T}(t) \Phi_{1} e(t)+e^{T}(t-\tau) \Phi_{2} e(t-\tau),
\end{aligned}
$$

where

$$
\begin{aligned}
& \Phi_{1}=-I_{N-1} \otimes\left[P A+A^{T} P+\tau R+\left(M_{1} \alpha+\mu L\right) \cdot I_{n}\right]-\left(\alpha^{-1}+\beta^{-1}\right)\left(I_{N-1} \otimes P\right)\left(I_{N-1} \otimes P\right), \\
& \Phi_{2}=M_{2} \alpha \cdot I_{n(N-1)}+\beta(\tilde{C} \otimes \Gamma)^{T}(\tilde{C} \otimes \Gamma) .
\end{aligned}
$$

We have

$$
\begin{aligned}
\Phi_{2}-\Phi_{1}= & M_{2} \alpha \cdot I_{n(N-1)}+\beta(\tilde{C} \otimes \Gamma)^{T}(\tilde{C} \otimes \Gamma) \\
& +I_{N-1} \otimes\left[P A+A^{T} P+\tau R+\left(M_{1} \alpha+\mu L\right) \cdot I_{n}\right] \\
& +\left(\alpha^{-1}+\beta^{-1}\right)\left(I_{N-1} \otimes P\right)\left(I_{N-1} \otimes P\right) \\
= & I_{N-1} \otimes\left[P A+A^{T} P+\tau R+\left(M_{1} \alpha+M_{2} \alpha+\mu L\right) \cdot I_{n}\right] \\
& +\beta(\tilde{C} \otimes \Gamma)^{T}(\tilde{C} \otimes \Gamma)+\left(\alpha^{-1}+\beta^{-1}\right)\left(I_{N-1} \otimes P\right)\left(I_{N-1} \otimes P\right) \\
\leq & I_{N-1} \otimes\left[P A+A^{T} P+\tau R+\left(M_{1} \alpha+M_{2} \alpha+\mu L\right) \cdot I_{n}\right] \\
& +\beta \lambda_{N}^{2} I_{N-1} \otimes\left(\Gamma^{T} \Gamma\right)+\left(\alpha^{-1}+\beta^{-1}\right)\left(I_{N-1} \otimes P\right)\left(I_{N-1} \otimes P\right) \\
= & I_{N-1} \otimes\left[P A+A^{T} P+\tau R+\left(M_{1} \alpha+M_{2} \alpha+\mu L\right) \cdot I_{n}\right. \\
& \left.+\beta \lambda_{N}^{2} \Gamma^{T} \Gamma+\left(\alpha^{-1}+\beta^{-1}\right) P^{2}\right] .
\end{aligned}
$$

By the Schur complete lemma [25], inequality (10) is equivalent to $\Phi_{2}<\Phi_{1}$. Thus, system (8) has almost sure asymptotic stability by Lemma 3 . Hence, complex network (1) without the control input has almost sure synchronization. The proof is completed. 
Remark 4 Inequalities (9) and (10) are linear matrix inequalities and can be easily solved by the LMI's toolbox in Matlab. Moreover, these inequalities only depend on the networks' parameters and the time delay. In addition, different from [12, 21], the inner-coupling matrix $\Gamma$ need not to be a diagonal matrix in this paper.

\subsection{Synchronization controller design}

In general, a complex network is not able to achieve synchronization without a control input. In this section, we will design appropriate controllers such that the closed-loop complex network has almost sure synchronization. Next, we introduce two methods, respectively.

(I) If we only control a part of nodes in the complex network. Without loss of generality, let the index number of controlled nodes be $i=2,3, \ldots, l+1,1 \leq l<N-1$, respectively. We take the controller as

$$
u_{i}(t)=-k_{i} e_{i}(t)
$$

then one gets

$$
U(t)=-\left[\mathscr{K}_{1} \otimes I_{n}\right] e(t)
$$

where $\mathscr{K}_{1}=\operatorname{diag}\{\underbrace{k_{2}, k_{3}, \ldots, k_{l+1}}_{l}, \underbrace{0, \ldots, 0}_{N-1-l}\}, k_{i}>0$ is the control gain to be determined. Thus, we obtain the closed-loop system

$$
\left\{\begin{aligned}
d e(t)= & {\left[\left(I_{N-1} \otimes A-\mathscr{K}_{1} \otimes I_{n}\right) e(t)+F(t, e(t), e(t-\tau))\right.} \\
& +(\tilde{C} \otimes \Gamma) e(t-\tau)] d t+G(e(t)) d w(t), \\
e(t)= & \Psi(t), \quad t \in[-\tau, 0] .
\end{aligned}\right.
$$

From Theorem 1, the following result is obtained.

Theorem 2 Suppose that assumptions ( $\mathrm{H} 1)$ and $(\mathrm{H} 2)$ hold. If there exist positive definite symmetric matrices $P, R \in R^{n \times n}$ and positive constants $\alpha>0, \beta>0, \mu>0, k_{i}>0$ $(i=2,3, \ldots, l)$, such that

$$
P<\mu I_{n}
$$

and

$$
\Theta_{2}=\left[\begin{array}{ccc}
\Theta_{2,11} & I_{N-1} \otimes P & I_{N-1} \otimes P \\
I_{N-1} \otimes P & -\alpha I_{n(N-1)} & 0 \\
I_{N-1} \otimes P & 0 & -\beta I_{n(N-1)}
\end{array}\right]<0
$$

hold, where

$$
\Theta_{2,11}=I_{N-1} \otimes\left[P A+A^{T} P+\tau R+\left(M_{1} \alpha+M_{2} \alpha+\mu L\right) \cdot I_{n}+\beta \lambda_{N}^{2} \Gamma^{T} \Gamma\right]-2 \mathscr{K}_{1} \otimes P,
$$

then complex network (1) has almost sure synchronization under the action of controller (19). 
As a special case of (I), if we only control one node, without loss of generality, assuming that the index number of controlled node is $i=1$. We take the controller as

$$
u_{1}(t)=k \sum_{i=2}^{N} e_{i}(t)
$$

thus

$$
U(t)=-k\left[\mathscr{L} \otimes I_{n}\right] e(t)
$$

where $\mathscr{L}$ is a $N-1$ order square matrix whose elements are $1, k>0$ is the control gain to be determined. Under the action of controller (23), we obtain the following closed-loop system:

$$
\left\{\begin{aligned}
d e(t)= & {\left[\left(I_{N-1} \otimes A-k \mathscr{L} \otimes I_{n}\right) e(t)+F(t, e(t), e(t-\tau))\right.} \\
& +(\tilde{C} \otimes \Gamma) e(t-\tau)] d t+G(e(t)) d w(t), \\
e(t)= & \Psi(t), \quad t \in[-\tau, 0] .
\end{aligned}\right.
$$

From Theorem 2, the following corollary is obtained.

Corollary 1 Suppose that assumptions (H1) and (H2) hold. If there exist positive definite symmetric matrices $P, R \in R^{n \times n}$ and positive constants $\alpha>0, \beta>0, \mu>0, k>0$, such that

$$
P<\mu I_{n}
$$

and

$$
\Theta_{3}=\left[\begin{array}{ccc}
\Theta_{3,11} & I_{N-1} \otimes P & I_{N-1} \otimes P \\
I_{N-1} \otimes P & -\alpha I_{n(N-1)} & 0 \\
I_{N-1} \otimes P & 0 & -\beta I_{n(N-1)}
\end{array}\right]<0
$$

hold, where

$$
\Theta_{3,11}=I_{N-1} \otimes\left[P A+A^{T} P+\tau R+\left(M_{1} \alpha+M_{2} \alpha+\mu L\right) \cdot I_{n}+\beta \lambda_{N}^{2} \Gamma^{T} \Gamma\right]-2 k \mathscr{L} \otimes P,
$$

then complex network (1) has almost sure synchronization under the action of controller (23).

(II) In order to obtain better control performance, we can use the following pinning adaptive controller. Let the index number of controlled nodes be $i=2,3, \ldots, l+1,1 \leq l<$ $N-1$, respectively. Taking the controller as

$$
u_{i}(t)=-k_{i}(t) e_{i}(t)
$$

and updated law as

$$
\dot{k}_{i}(t)=\delta\left\|e_{i}(t)\right\|^{2}
$$


then one gets

$$
U(t)=-\left[\mathscr{K}(t) \otimes I_{n}\right] e(t),
$$

where $\mathscr{K}(t)=\operatorname{diag}\{\underbrace{k_{2}(t), k_{3}(t), \ldots, k_{l+1}(t)}_{l}, \underbrace{0, \ldots, 0}_{N-1-l}\}, \delta>0$ is any positive constant. Thus, we obtain the following closed-loop system:

$$
\left\{\begin{aligned}
& \operatorname{de}(t)=\left\{\left[I_{N-1} \otimes A-\mathscr{K}(t) \otimes I_{n}\right] e(t)+F(t, e(t), e(t-\tau))\right. \\
&+(\tilde{C} \otimes \Gamma) e(t-\tau)\} d t+G(e(t)) d w(t), \\
& \dot{\mathscr{K}}(t)= \operatorname{diag}\{\delta\left\|e_{2}(t)\right\|^{2}, \delta\left\|e_{3}(t)\right\|^{2}, \ldots, \delta\left\|e_{l+1}(t)\right\|^{2}, \underbrace{0, \ldots, 0}_{N-1-l}\}, \\
& e(t)=\Psi(t), \quad t \in[-\tau, 0] .
\end{aligned}\right.
$$

Similar to Theorem 1, we obtain the following result.

Theorem 3 Suppose that assumptions $(\mathrm{H} 1)$ and $(\mathrm{H} 2)$ hold. If there exist positive definite symmetric matrices $P, R \in R^{n \times n}$ and positive constants $\alpha>0, \beta>0, \mu>0, k^{*}>0$, such that

$$
P<\mu I_{n}
$$

and

$$
\Theta_{4}=\left[\begin{array}{ccc}
\Theta_{4,11} & I_{N-1} \otimes P & I_{N-1} \otimes P \\
I_{N-1} \otimes P & -\alpha I_{n(N-1)} & 0 \\
I_{N-1} \otimes P & 0 & -\beta I_{n(N-1)}
\end{array}\right]<0
$$

hold, where

$$
\Theta_{4,11}=I_{N-1} \otimes\left[P A+A^{T} P+\tau R+\left(M_{1} \alpha+M_{2} \alpha+\mu L\right) \cdot I_{n}+\beta \lambda_{N}^{2} \Gamma^{T} \Gamma\right]-2 \mathscr{K}_{2} \otimes I_{n},
$$

$\mathscr{K}_{2}=\operatorname{diag}\{\underbrace{k^{*}, k^{*}, \ldots, k^{*}}_{l}, \underbrace{0, \ldots, 0}_{N-1-l}\}$, then complex network (1) has almost sure synchronization under the action of pinning adaptive controller (28).

Proof Choose the Lyapunov function as

$$
V_{1}(e(t))=V(e(t))+\frac{1}{\delta} \sum_{i=2}^{l}\left(k_{i}(t)-k^{*}\right)^{2},
$$

where $V(e(t))$ is the same as in Theorem 1 . After some necessary computation, we find that the differential of $V_{1}(e(t))$ along the state trajectories of system (29) satisfies

$$
\mathcal{L} V_{1}(e(t))<\mathcal{L} V(e(t))-2 e^{T}(t) \mathscr{K}_{2} e(t)
$$

As inequalities (30) and (31) hold, $\mathcal{L} V_{1}(e(t))<0$. Hence, complex network (1) has almost sure synchronization under the action of controller (28). The proof is completed. 
Remark 5 It is worth mentioning that the pinning control is one of important control methods for complex network and has been studied in some literature such as [16-18]. Obviously, this method also suits for the case of controlling every node in complex network. Specially, while controlling $N-1$ nodes, inequalities (30) and (31) must exist feasible solutions because $k^{*}$ can be chosen sufficiently large, which shows that complex network (1) could achieve synchronization under the action of adaptive controller (28). Furthermore, the synchronization speed can be adjusted by tuning $\delta$.

\section{A numerical example}

In this section, we provide a numerical example to illustrate the effectiveness of our proposed methods.

Example 1 Consider the following dynamical system:

$$
\left\{\begin{array}{l}
\dot{z}_{1}(t)=10\left[z_{2}(t)-z_{1}(t)-f_{1}\left(z_{1}(t), z_{1}(t-\tau)\right)\right] \\
\dot{z}_{2}(t)=z_{1}(t)-z_{2}(t)+z_{3}(t) \\
\dot{z}_{3}(t)=-15 z_{2}(t)-0.0385 z_{3}(t)
\end{array}\right.
$$

where $f_{1}\left(z_{1}(t), z_{1}(t-\tau)\right)=b z_{1}(t)+0.5(a-b)\left(\left|z_{1}(t)+1\right|-\left|z_{1}(t-\tau)-1\right|\right), a, b$ are two constants. System (34) can be written in vector form,

$$
\dot{z}(t)=A z(t)+f(z(t), z(t-\tau))
$$

where

$$
\begin{aligned}
& z(t)=\left(z_{1}(t), z_{2}(t), z_{3}(t)\right)^{T}, \\
& A=\left[\begin{array}{ccc}
-10 & 10 & 0 \\
1 & -1 & 1 \\
0 & -15 & -0.038
\end{array}\right], \\
& f(z(t), z(t-\tau))=\left(-10 f_{1}\left(z_{1}(t), z_{1}(t-\tau)\right), 0,0\right)^{T} .
\end{aligned}
$$

Since

$$
\begin{aligned}
\|f(z(t), z(t-\tau))-f(\hat{z}(t), \hat{z}(t-\tau))\|_{2} \\
=10\left|f_{1}\left(z_{1}(t), z_{1}(t-\tau)\right)-f_{1}\left(\hat{z}_{1}(t), \hat{z}_{1}(t-\tau)\right)\right| \\
\leq 10\left(|b|+\frac{1}{2}|a-b|\right) \cdot\left|z_{1}(t)-\hat{z}_{1}(t)\right| \\
\quad+5|a-b| \cdot\left|z_{1}(t-\tau)-\hat{z}_{1}(t-\tau)\right| \\
\leq 10\left(|b|+\frac{1}{2}|a-b|\right) \cdot\|z(t)-\hat{z}(t)\|_{2} \\
\quad+5|a-b| \cdot\|z(t-\tau)-\hat{z}(t-\tau)\|_{2}
\end{aligned}
$$

for any $z(t), \hat{z}(t) \in R^{3}, f(z(t))$ satisfies assumption (H1). When $\tau=0$, system (34) is the Chua dynamical system. 
Consider complex network (1) with ten nodes $(N=10)$ and take system (34) as each node. Other parameters are as follows:

$$
\begin{aligned}
C & =\left[\begin{array}{cccccccccc}
-7 & 1 & 0 & 1 & 1 & 1 & 0 & 1 & 1 & 1 \\
1 & -5 & 1 & 0 & 1 & 0 & 0 & 1 & 0 & 1 \\
0 & 1 & -6 & 0 & 1 & 1 & 1 & 0 & 1 & 1 \\
1 & 0 & 0 & -5 & 1 & 1 & 0 & 1 & 0 & 1 \\
1 & 1 & 1 & 1 & -7 & 0 & 1 & 1 & 0 & 1 \\
1 & 0 & 1 & 1 & 0 & -6 & 1 & 1 & 1 & 0 \\
0 & 0 & 1 & 0 & 1 & 1 & -5 & 0 & 1 & 1 \\
1 & 1 & 0 & 1 & 1 & 1 & 0 & -7 & 1 & 1 \\
1 & 0 & 1 & 0 & 0 & 1 & 1 & 1 & -5 & 0 \\
1 & 1 & 1 & 1 & 1 & 0 & 1 & 1 & 0 & -7
\end{array}\right], \\
\Gamma & =\left[\begin{array}{ccc}
0.2 & -0.3 & 1 \\
-1 & 0.5 & -0.4 \\
0.2 & -0.9 & 0.7
\end{array}\right],
\end{aligned}
$$

Table 1 The solutions of the inequalities in Theorems 1-3 and Corollary 1 with the parameters such as Example 1

\begin{tabular}{lllllll}
\hline & $\boldsymbol{P}$ & $\boldsymbol{R}$ & $\boldsymbol{\alpha}$ & $\boldsymbol{\beta}$ & $\boldsymbol{\mu}$ & \\
\hline Theorem 1 & - & - & - & - & - & - \\
Corollary 1 & - & - & - & - & - & - \\
Theorem 2 & $P_{3}$ & $R_{3}$ & 0.1226 & 0.1474 & 1.4654 & $I=9, k=50$ \\
Theorem 3 & $P_{4}$ & $R_{4}$ & 0.2079 & 0.0403 & 0.6772 & $I=9, k^{*}=30$ \\
\hline
\end{tabular}

Figure 1 State trajectories of the complex network under the action of pinning controller (19). (a) State trajectories of every node; (b) error state trajectories of every node with the first node.

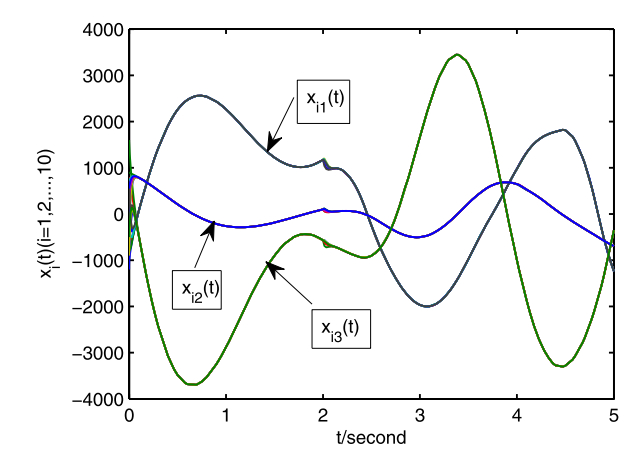

(a)

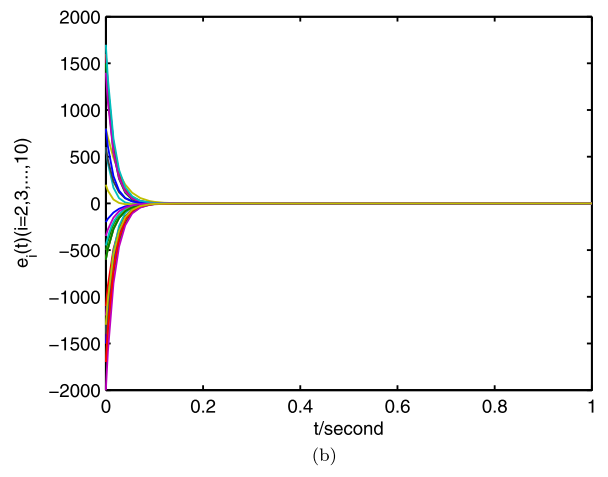


Figure 2 State trajectories of the complex network under the action of pinning adaptive controller (28). (a) State trajectories of every node; (b) error state trajectories of every node with the first node; (c) state trajectories of updated law (27) with $\delta=0.02$.
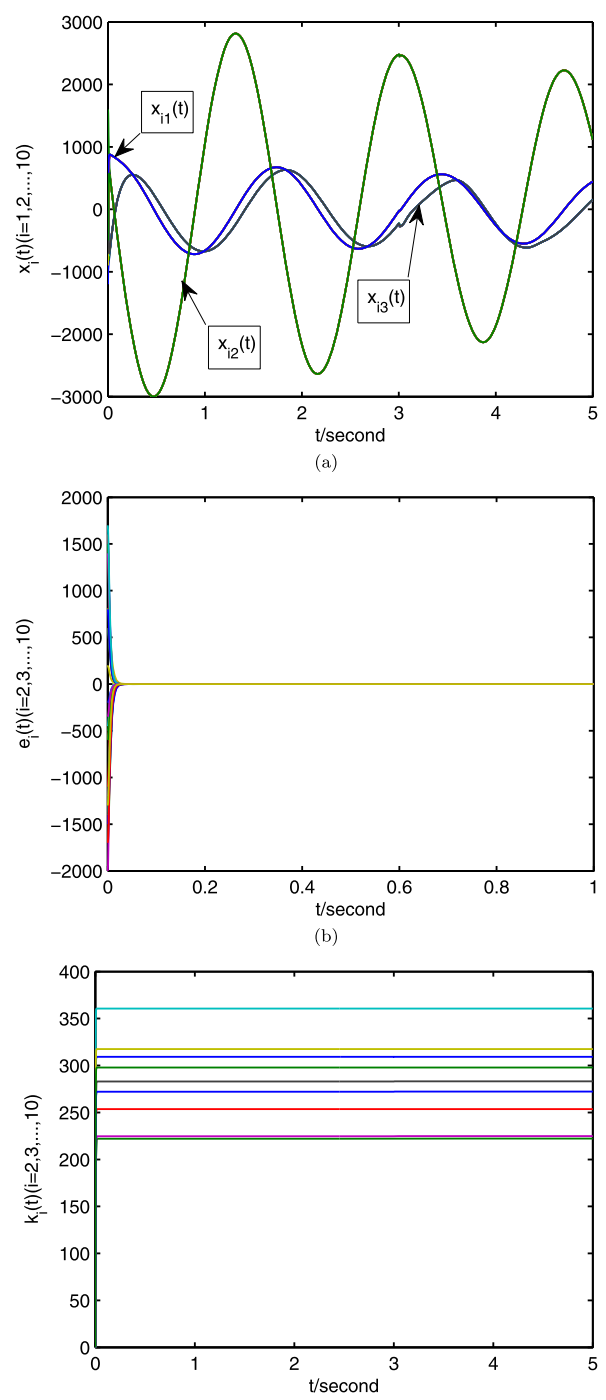

$a=-1.31, b=-0.75, \tau=2$. The noise intensity is $g\left(x_{i}(t)\right)=\tanh \left(x_{i}(t)\right)$ for $1 \leq i \leq 10$. It is easy to verify that assumption (H2) holds when setting $L=1$. By the LMI's toolbox in Matlab, we obtain the feasible solutions of the inequalities in Theorems 1-3 and Corollary 1 shown in Table 1, respectively, where

$$
\begin{aligned}
& P_{3}=\left[\begin{array}{ccc}
0.3383 & -0.0783 & 0.1283 \\
-0.0783 & 0.4719 & -0.2695 \\
0.1283 & -0.2695 & 0.6098
\end{array}\right], \quad R_{3}=\left[\begin{array}{ccc}
0.8439 & 0.0067 & -0.1303 \\
0.0067 & 0.6222 & 0.3037 \\
-0.1303 & 0.3037 & 0.6121
\end{array}\right] \text {, } \\
& P_{4}=\left[\begin{array}{ccc}
0.0307 & 0.0102 & 0.0108 \\
0.0102 & 0.0205 & -0.0084 \\
0.0108 & -0.0084 & 0.0338
\end{array}\right], \quad R_{4}=\left[\begin{array}{ccc}
4.8350 & 1.6149 & -1.7028 \\
1.6149 & 4.0109 & 2.4636 \\
-1.7028 & 2.4636 & 3.5952
\end{array}\right] \text {. }
\end{aligned}
$$

From Table 1, we can see that the inequalities in Theorem 1 and Corollary 1 are infeasible and as regards the inequalities in Theorem 2 and Theorem 3 there exist feasible solutions, which implies that the complex network could not synchronize with each other if lacking 
control input or only controlling one node, and they could synchronize well if utilizing pinning controller or pinning adaptive controller. Our simulating computation, we depict in five figures. In particular, Figure 1 is the state trajectories of complex network and its error system under the action of pinning controller (19), these figures show that all the nodes synchronize well. Figure 2 is the state trajectories of complex network, its error system and updated laws under the action of pinning adaptive controller (28), respectively, which shows that all the nodes have synchronization.

\section{Conclusions}

This paper has investigated the almost sure synchronization control problem for a class of stochastic delayed complex networks based on the stochastic differential equation theory. Some synchronization criteria and two kinds of pinning controllers have been proposed. These results reflect the relation of synchronization to the parameters of complex networks. A numerical example has shown that our method is effective.

This paper investigated the almost sure synchronization control other than synchronization in mean square of complex networks, and the obtained results may be appropriate for the synchronization of complex networks with the target node unknown. Specially, the results obtained only depend on the complex network's parameters.

Competing interests

The authors declare that they have no competing interests.

Authors' contributions

The authors contributed equally to this article. The authors read and approved this article.

\section{Author details}

${ }^{1}$ College of Fundamental Studies, Shanghai University of Engineering Science, Longteng Road, Shanghai, P.R. China.

${ }^{2}$ College of Information Sciences and Technology, Donghua University, Renmin Road, Shanghai, P.R. China.

\section{Acknowledgements}

This work was supported by the National Natural Science Foundation of China (11602134), the Shanghai Natural Science Foundation (16ZR1413700), and the Natural Science Foundation of Shanghai University of Engineering Science (nhrc-2015-06).

Received: 21 May 2016 Accepted: 25 October 2016 Published online: 29 November 2016

References

1. Jeong, H, Tombor, B, Albert, R, Oltvai, ZN, Barabasi, AL: The large-scale organization of metabolic networks. Nature 407, 651-654 (2000)

2. Bennett, $M$, Zukin, B: Electrical coupling and neuronal synchronization in the mammalian brain. Neuron 41, 495-511 (2004)

3. Strogatz, SH: Exploring complex networks. Nature 410, 268-276 (2001)

4. Li, ZK, Duan, ZS, Chen, GR, Huang, L: Consensus of multi-agent systems and synchronization of complex networks: a unified viewpoint. IEEE Trans. Circuits Syst. I, Regul. Pap. 57, 213-224 (2010)

5. Yahyazadeh, M, Noei, AR, Ghaderi, R: Synchronization of chaotic systems with known and unknown parameters using a modified active sliding mode control. ISA Trans. 50, 262-267 (2011)

6. Lu, WL, Chen, TP: Global synchronization of discrete-time dynamical network with a directed graph. IEEE Trans. Circuits Syst. II, Express Briefs 54, 136-140 (2007)

7. Shen, $B$, Wang, ZD, Liu, XH: Bounded $H_{\infty}$ synchronization and state estimation for discrete time-varying stochastic complex networks over a finite horizon. IEEE Trans. Neural Netw. 22, 145-156 (2011)

8. Wang, JL, Wu, HN, Huang, TW: Passivity-based synchronization of a class of complex dynamical networks with time-varying delay. Automatica 56, 105-112 (2015)

9. Jin, T, Li, WX, Feng, JY: Outer synchronization of stochastic complex networks with time-varying delay. Adv. Differ. Equ. 2015, 359 (2015)

10. Wang, L, Qian, W, Wang, QG: Exponential synchronization in complex networks with a single coupling delay. J. Franklin Inst. 350, 1406-1423 (2013)

11. Jin, YG, Zhong, SM: Function projective synchronization in complex networks with switching topology and stochastic effects. Appl. Math. Comput. 259, 730-740 (2015)

12. Tang, Y, Leung, SYS, Wong, WK, Fang, JA: Impulsive pinning synchronization of stochastic discrete-time networks. Neurocomputing 73, 2132-2139 (2010) 
13. Yao, J, Wang, HO, Guan, ZH, Xu, WS: Passive stability and synchronization of complex spatio-temporal switching networks with time delays. Automatica 45, 1721-1728 (2009)

14. Chen, WH, Jiang, ZY, Lu, XM, Luo, SX: $H_{\infty}$ synchronization for complex dynamical networks with coupling delays using distributed impulsive control. Nonlinear Anal. Hybrid Syst. 17, 111-127 (2015)

15. Li, CJ, Yu, WW, Huang, TW: Impulsive synchronization schemes of stochastic complex networks with switching topology: average time approach. Neural Netw. 54, 85-94 (2014)

16. Wu, YY, Wei, W, Li, GY, Xiang, J: Pinning control of uncertain complex networks to a homogeneous orbit. IEEE Trans. Circuits Syst. II 56, 235-239 (2009)

17. Gong, $\mathrm{XL}, \mathrm{Wu}, \mathrm{ZY}$ : Adaptive pinning impulsive synchronization of dynamical networks with time-varying delay. Adv. Differ. Equ. 2015, 240 (2015)

18. Zhou, J, Wu, QJ, Xiang, L: Pinning complex delayed dynamical networks by a single impulsive controller. IEEE Trans. Circuits Syst. I, Regul. Pap. 58, 2882-2893 (2011)

19. Yang, XS, Cao, JD, Lu, JQ: Stochastic synchronization of complex networks with nonidentical nodes via hybrid adaptive and impulsive control. IEEE Trans. Circuits Syst. I, Regul. Pap. 59, 371-384 (2012)

20. Wu, ZY, Wang, HJ: Impulsive pinning synchronization of discrete-time network. Adv. Differ. Equ. 2016, 36 (2016)

21. Yu, WW, DeLellis, P, Chen, GR, Bernardo, M, Kurths, J: Distributed adaptive control of synchronization in complex networks. IEEE Trans. Autom. Control 57, 2153-2158 (2012)

22. Zhu, QX, Cao, JD: Adaptive synchronization of chaotic Cohen-Crossberg neural networks with mixed time delays. Nonlinear Dyn. 61, 517-534 (2010)

23. Mahbashi, GA, Noorani, MS, Bakar, SA, Sawalha, MA: Adaptive projective lag synchronization of uncertain complex dynamical networks with delay coupling. Adv. Differ. Equ. 2015, 356 (2015)

24. Song, B, Park, JH, Wu, ZG, Zhang, Y: Global synchronization of stochastic delayed complex networks. Nonlinear Dyn. 70, 2389-2399 (2012)

25. Rakkiyappan, R, Dharani, S, Zhu, QX: Stochastic sampled-data $H_{\infty}$ synchronization of coupled neutral-type delay partial differential systems. J. Franklin Inst. 352, 4480-4502 (2015)

26. Zhou, WN, Wang, TB, Mou, JP, Fang, JA: Mean square exponential synchronization in Lagrange sense for uncertain complex dynamical networks. J. Franklin Inst. 349, 1267-1282 (2012)

27. Yang, XS, Cao, JD, Lu, JQ: Synchronization of delayed complex dynamical networks with impulsive and stochastic effects. Nonlinear Anal., Real World Appl. 12, 2252-2266 (2011)

28. $\mathrm{Hu}, \mathrm{L}, \mathrm{Mao}, \mathrm{XR}$ : Almost sure exponential stabilization of stochastic systems by state-feedback control. Automatica 44, 465-471 (2008)

29. Zhu, QX, Li, XD: Exponential and almost sure exponential stability of stochastic fuzzy delayed Cohen-Grossberg neural networks. Fuzzy Sets Syst. 203, 74-94 (2012)

30. Mao, XR, Shen, Y, Yuan, CG: Almost surely asymptotic stability of neutral stochastic differential delay equations with Markovian switching. Stoch. Process. Appl. 118, 1385-1406 (2008)

31. Zhu, QX, Cao, JD: Adaptive synchronization under almost every initial data for stochastic neural networks with time-varying delays and distributed delays. Commun. Nonlinear Sci. Numer. Simul. 16, $2139-2159$ (2011)

32. Lu, JQ, Daniel, WCH: Globally exponential synchronization and synchronizability for general dynamical networks. IEEE Trans. Syst. Man Cybern. 40, 350-361 (2010)

33. Mao, XR: A note on the LaSalle-type theorems for stochastic differential delay equations. J. Math. Anal. Appl. 268 125-142 (2002)

34. Horn, RA, Johnson, CR: Matrix Analysis. Cambridge University Press, New York (1985)

\section{Submit your manuscript to a SpringerOpen ${ }^{\circ}$ journal and benefit from:}

- Convenient online submission

Rigorous peer review

- Immediate publication on acceptance

- Open access: articles freely available online

- High visibility within the field

- Retaining the copyright to your article 